\title{
Dynamics and Transport in Random Antiferromagnetic Spin Chains
}

\author{
Kedar Damle, ${ }^{1,2}$ Olexei Motrunich, ${ }^{1}$ and David A. Huse ${ }^{1}$ \\ ${ }^{1}$ Department of Physics, Princeton University, Princeton, New Jersey 08544 \\ ${ }^{2}$ Department of Electrical Engineering, Princeton University, Princeton, New Jersey 08544
}

(Received 15 November 1999)

\begin{abstract}
We present the first results on the low-frequency dynamical and transport properties of random antiferromagnetic spin chains at low temperature $(T)$. We obtain the momentum and frequency dependent dynamic structure factor in the random singlet (RS) phases of both spin- $1 / 2$ and spin- 1 chains, as well as in the random dimer phase of spin-1/2 chains. We also show that the RS phases are unusual "spin-metals" with divergent low-frequency conductivity at $T=0$, and follow the spin conductivity through "metalinsulator" transitions tuned by the strength of dimerization or Ising anisotropy in the spin- $1 / 2$ case and by the strength of disorder in the spin- 1 case.
\end{abstract}

PACS numbers: 75.10.Jm, 71.30.+h, 75.50.Ee, 78.70.Nx

Low-dimensional quantum spin systems in the presence of disorder are a fascinating laboratory for the study of the interplay between quantum effects, strong correlations and disorder. This is, in part, because they are often very sensitive to disorder, and display dramatic effects due to small amounts of disorder (especially in one dimension). The question of disorder effects is thus of considerable experimental importance in such cases. Moreover, a great deal is now known about the effects of strong correlations and quantum fluctuations in pure one- and two-dimensional spin systems [1], allowing one to focus on the new features introduced by disorder.

Random antiferromagnetic spin- $1 / 2$ chain compounds are interesting, experimentally realizable examples [2,3] of such systems. Theoretical work has led to the prediction that even a small amount of disorder can cause these systems to have an extremely unusual "random singlet" (RS) ground state for a range of parameter values [4-6]. In this RS state, the interplay of disorder and quantum mechanics locks each spin into a singlet pair with another spin; the two spins in a given singlet pair can have arbitrarily large spatial separation. A similar RS state is also predicted in the spin-1 Heisenberg antiferromagnetic chain for sufficiently strong randomness in the bonds [6,7]. The theory also yields unusual results for the low-temperature thermodynamics and some ground-state correlators in the RS states.

In this Letter, we report on the first theoretical study of the dynamical and transport properties of these systems; the primary motivation is of course to understand the dynamics and transport when quantum interference, strong correlations, and disorder are all simultaneously important. We focus here on the momentum and frequency dependent dynamical structure factor $S(k, \omega)$ and the dynamical spin conductivity. The former is directly probed by inelastic neutron scattering (INS) experiments on these compounds, while the latter is of considerable theoretical interest as it contains information about the nature of any localization phenomena, or lack thereof, in these strongly correlated disordered systems.
In particular, we calculate the low-temperature $S(k, \omega)$ at low frequencies in the RS states of spin- $1 / 2$ and spin-1 chains as well as in the "random dimer" (RD) phase of spin-1/2 chains that is predicted [8] in the presence of weak enforced bond dimerization and strong disorder. Our results lead us to expect some very unusual features in the INS cross section as a function of wave vector transfer $k$ (for fixed, small energy transfer $\omega \gtrsim T$ ), especially in the RD phase. For the conductivity, we calculate the real part of the low-frequency dynamical spin conductivity $\sigma^{\prime}(\omega)$ at low temperatures and show that the RS phases of both spin- $1 / 2$ and spin- 1 chains are actually unusual "spinmetals" with a $\sigma^{\prime}(\omega)$ that diverges for low $\omega$ at $T=0$. We also follow $\sigma^{\prime}(\omega)$ across novel "metal-insulator" transitions to insulating states; these are accessed in the spin- $1 / 2$ case by turning on dimerization (leading to a RD phase) or by increasing the Ising anisotropy above a threshold, and are accessed in the spin-1 Heisenberg case by reducing the disorder below a critical value. Thus, we are able to obtain a wealth of reliable information about the unusual dynamics and transport in these strongly correlated random quantum systems. We emphasize that all our results are exact in the low-frequency limit at $T=0$, and they continue to be valid for $T \neq 0$ as long as $T \lesssim \omega$ (in some cases, we are also able to access frequencies $\omega<T$ [9]).

In the spin- $1 / 2$ case, the specific problem we consider is the random $X X Z$ Hamiltonian, which describes the lowenergy (magnetic) dynamics of insulating antiferromagnetic spin-1/2 chain compounds [2,3] with chemical disorder that affects the bond strengths:

$$
\mathcal{H}_{X X Z}=\sum_{j}\left[J_{j}^{\perp}\left(s_{j}^{x} s_{j+1}^{x}+s_{j}^{y} s_{j+1}^{y}\right)+J_{j}^{z} s_{j}^{z} s_{j+1}^{z}\right],
$$

where $\vec{s}_{j}$ are spin-1/2 operators at lattice sites $j$ separated by spacing $a$, and both $J_{j}^{\perp}$ and $J_{j}^{z}$ are random positive exchange energies with a joint probability distribution $P_{0}\left(J^{\perp}, J^{z}\right)$. Detailed information on the nature of the excitations in such systems is encoded in the dynamical structure factor $S^{\alpha \beta}(k, \omega)$ (where $\alpha \beta \equiv+-$ or $\alpha \beta \equiv z z$ ) with the $T=0$ spectral representation 


$$
S^{\alpha \beta}(k, \omega)=\frac{1}{L} \sum_{m}\left|\left\langle m\left|\sum_{j=1}^{L} e^{i k x_{j}} s_{j}^{\alpha}\right| 0\right\rangle\right|^{2} \delta\left(\omega-E_{m}\right),
$$

where $s^{ \pm} \equiv s^{x} \pm i s^{y}$ and $\{|m\rangle\}$ denote the exact eigenstates of the system with energies $E_{m}(|0\rangle$ is the ground state, with $E_{0}=0$ ).

As mentioned earlier, we characterize transport in terms of the dynamical conductivity $\sigma(\omega)$. The real part $\sigma^{\prime}(\omega)$ of $\sigma(\omega)$ is defined by the relation $P(\omega)=\sigma^{\prime}(\omega)|\nabla H|^{2}(\omega)$, where $P(\omega)$ is the power absorbed per unit volume by the system when a uniform magnetic field gradient $\nabla H(\omega)$ (where the field $H$ always points in the $z$ direction) oscillating at frequency $\omega$ is applied along the length of the chain. From standard linear response theory, we have the following Kubo formula for $\sigma^{\prime}(\omega)$ at $T=0$ :

$$
\sigma^{\prime}(\omega)=\frac{1}{\omega L} \sum_{m}\left|\left\langle m\left|\sum_{j=1}^{L} \tau_{j}\right| 0\right\rangle\right|^{2} \delta\left(\omega-E_{m}\right),
$$

where $\tau_{j}=-i J_{j}^{\perp}\left(s_{j+1}^{+} s_{j}^{-}-s_{j}^{+} s_{j+1}^{-}\right) / 2$ is the current operator on link $j$ that transfers one unit of the $z$ component of the spin from one site to the next, and the frequency $\omega$ is taken positive for notational convenience. Note that both $S^{\alpha \beta}(k, \omega)$ and $\sigma^{\prime}(\omega)$, as defined here, are self-averaging in the thermodynamic limit.

Randomness in the bonds is a relevant perturbation [5] for the pure $X X Z$ chain when $0 \leq J^{z} / J^{\perp} \leq 1$; any amount of disorder is thus expected to drive the system to strong disorder. In this regime, the system can be treated by a strong-randomness renormalization group (RG) that proceeds as follows $[4,6,10]$ : We look for the bond with the largest $J^{\perp}$ in the chain, say, $J_{23}^{\perp}$ between spins 2 and 3 - this sets the energy cutoff $\Omega=\max \left\{J_{j}^{\perp}\right\}$. We first solve the corresponding two spin problem (the neighboring bonds are introduced later as perturbations). As long as the $J^{z}$ are not large compared to the $J^{\perp}$, the ground state of the two-spin problem will always be a singlet separated by a large gap from the triplet excited states. We can then trade our original Hamiltonian in for another Hamiltonian (determined perturbatively in the ratio of the neighboring bonds to the strongest bond) which acts on a truncated Hilbert space with the two sites connected by the "strong" bond removed. To leading order, this procedure renormalizes the Hamiltonian $\mathcal{H}_{4 \text { sites }}=\sum_{j=1}^{3} J_{j}^{\perp}\left(s_{j}^{x} s_{j+1}^{x}+s_{j}^{y} s_{j+1}^{y}\right)+J_{j}^{z} s_{j}^{z} s_{j+1}^{z}$ to $\tilde{\mathcal{H}}_{14}=\tilde{J}_{1}^{\perp}\left(s_{1}^{x} s_{4}^{x}+s_{1}^{y} s_{4}^{y}\right)+\tilde{J}_{1}^{z} s_{1}^{z} s_{4}^{z}$ with $\tilde{J}_{1}^{\perp}=J_{1}^{\perp} J_{3}^{\perp} /$ $\left(J_{2}^{\perp}+J_{2}^{z}\right)$ and $\tilde{J}_{1}^{z}=J_{1}^{z} J_{3}^{z} / 2 J_{2}^{\perp}$ [6]. Note that the new bond has length $\tilde{l}_{1}=l_{1}+l_{2}+l_{3}$. This procedure, if it remains valid upon iteration, thus ultimately leads to a ground state of singlet pairs, with pairs formed over long distances held together by correspondingly weak bonds; this is the RS state alluded to earlier.

A complete understanding of the possible states thus requires an analysis of the effects of iterating the basic RG procedure. Such an analysis was performed in Ref. [6], leading to the following conclusions: If the $J^{z}$ dominate over the $J^{\perp}$, this procedure rapidly becomes invalid and the ground state actually has Ising antiferromagnetic (IAF) order. In all other cases, the ground state is a random singlet state. The low-energy (with energy cutoff $\Omega \ll \Omega_{c}$, where $\Omega_{c}$ is the microscopic cutoff) effective theory in these cases is written in terms of the $n(\Omega) L$ "surviving" spin variables (the bond-length $l$ between successive surviving sites is now a random quantity). When the $J^{\perp}$ dominate, this effective Hamiltonian has all $J_{j}^{z}=0$, and $J^{\perp}$ and $l$ drawn from a universal joint probability distribution $\mathcal{P}\left(J^{\perp}, l \mid \Omega\right)$ characteristic of the " $X X$ random singlet fixed point" $(X X-\mathrm{RS})$ of the RG. Between the IAF phase and this $X X$-RS phase lie two kinds of critical points. If the initial problem has full Heisenberg symmetry $\left(J^{z}=J^{\perp}\right.$ for each bond), the low-energy effective Hamiltonian preserves this symmetry and has bonds strengths and lengths drawn from the same probability distribution $\mathcal{P}(J, l \mid \Omega)$. In the RG language, the Heisenberg system is controlled by the $X X X$-RS fixed point. Finally, in this language, the generic critical state is controlled by the XXZC-RS fixed point-the lowenergy effective theory has bonds and lengths drawn from a fixed point distribution $\mathcal{P}_{1}\left(J^{\perp}, J^{z}, l \mid \Omega\right)$ with the property $\int d J^{z} \mathcal{P}_{1}=\mathcal{P}\left(J^{\perp}, l \mid \Omega\right)$. The probability distributions $\mathcal{P}$ and $\mathcal{P}_{1}$ become infinitely broad as $\Omega \rightarrow 0$; this implies that the RG becomes asymptotically exact at low energies and, in particular, predicts the ground-state properties and low-temperature thermodynamics correctly. Note that, in all of the above, we have suppressed nonuniversal scale factors multiplying the arguments of the functions $n$ and $\mathcal{P}$; these scaling functions however remain the same for all systems that flow to any of the RS fixed points.

To use the foregoing for the calculation of dynamical or transport properties, we need to explicitly keep track of the renormalization of the operators that enter spectral sums such as (2) and (3). We illustrate our general approach [9] by first calculating the $T=0$ dynamical conductivity at asymptotically low frequencies. The first step is to work out the rules that govern the renormalization of the current operators. Assume, once again, that $J_{23}^{\perp}$ is the strongest bond. We wish to work out perturbatively the renormalized operators $\tilde{\tau}_{1 / 2 / 3}$, which we trade in $\tau_{1 / 2 / 3}$ for, when we freeze spins 2 and 3 in their singlet ground state (the other current operators to the left and right of this segment of our system are left unchanged to leading order by the renormalization). Now, note that these other operators have an overall scale factor in them that is nothing but the corresponding $J^{\perp}$. In order to be consistent, we clearly need to work out $\tilde{\tau}_{1 / 2 / 3}$ correct to $O\left(\tilde{J}_{1}^{\perp}\right)$ by adding the effects of virtual fluctuations to the projection of $\tau_{1 / 2 / 3}$ into the singlet subspace [11]. An explicit calculation [9] gives the simple result: $2 \tilde{\tau}_{2}=2 \tilde{\tau}_{1 / 3}=-i \tilde{J}_{1}^{\perp}\left(s_{4}^{+} s_{1}^{-}-s_{1}^{+} s_{4}^{-}\right)$. Thus, all three operators renormalize to the same operator, 
which we will denote henceforth by $\tilde{\tau}_{1}$ for consistency of notation.

As we carry out the RG and reduce the energy cutoff, the above result implies that $\sum_{j} \tau_{j}$ renormalizes to $\tilde{\sum}_{j} \tilde{l}_{j} \tilde{\tau}_{j}$, where $j$ now labels the $n_{\Omega} L$ sites of the renormalized lattice at cutoff scale $\Omega$, and the $\tilde{l}_{j}$ are the lengths of the renormalized bonds in this problem. With this in hand, we run the RG until the cutoff is reduced to $\Omega_{\omega}=\omega$ (note that the operator $\tilde{\tau}_{j}$ linking two sites connected by a strong bond $\left\{\tilde{J}_{j}^{\perp}, \tilde{J}_{j}^{z}\right\}$ promotes the corresponding pair of spins from their singlet ground state to the triplet state $\left|t_{0}\right\rangle$ with $m_{z}=0$, which is separated from the ground state by precisely $\tilde{J}_{j}^{\perp}$ ) and rewrite (3) as

$$
\sigma^{\prime}(\omega)=\frac{1}{\omega L} \sum_{m}\left|\left\langle m\left|\tilde{\sum}_{j} \tilde{l}_{j} \tilde{\tau}_{j}\right| 0\right\rangle\right|^{2} \delta\left(\omega-\tilde{E}_{m}\right),
$$

where the tildes are a reminder of the fact that this spectral sum now refers to the new Hamiltonian with cutoff $\omega$ which has only $n_{\omega} L$ sites (the couplings and bond lengths in this problem are of course drawn from the probability distribution characteristic of the fixed point to which the system flows in the low-energy limit).

We now have to calculate the spectral sum (4) in this new problem. The following crucial observation allows us to do this: At the next step of the RG, one would have looked for all of the bonds in this renormalized problem that have $J^{\perp}$ in the range $\left(\Omega_{\omega}, \Omega_{\omega}-d \Omega\right)$ and formed singlets out of the corresponding pairs of spins. The states with $\tilde{E}_{m}=\omega$ that give the dominant contribution to the spectral sum (4) correspond precisely [12] to promoting any one of these pairs to the triplet state $\left|t_{0}\right\rangle$ under the action of the current operator living on the corresponding bond. The matrix element for this transition is just $\tilde{l} \omega / 2$, where $\tilde{l}$ is the length of the bond in question. In the thermodynamic limit, we thus have

$$
\begin{aligned}
\sigma^{\prime}(\omega) & \sim \frac{n_{\Omega_{\omega}}}{\omega} \int d l d J^{\perp} \omega^{2} l^{2} \mathcal{P}\left(J^{\perp}, l \mid \Omega_{\omega}\right) \delta\left(\omega-J^{\perp}\right) \\
& \sim \ln \left(\Omega_{c} / \omega\right)
\end{aligned}
$$

the last line is the leading behavior for $\omega \ll \Omega_{c}$ obtained by using the results of Ref. [6] for $n_{\Omega}$ and $\mathcal{P}$. Notice that this analysis holds equally well at all three RS fixed points.

Thus $\sigma^{\prime}(\omega)$ diverges logarithmically for small enough $\omega$ in the unusual "spin-metal" phase controlled by the $X X$ fixed point [13] as well as at the critical point separating this phase from the insulating phase with IAF order in the ground state. Close to the transition on the insulating side, $\sigma^{\prime}(\omega)$ is suppressed below a pseudogap energy $E_{g} \ll \Omega_{c}$ which goes to zero as the transition is approached (for $E_{g} \ll \omega \ll \Omega_{c}, \sigma^{\prime}$ continues to diverge logarithmically as at the critical point). The dominant contributions for $\omega \ll E_{g}$ come from Griffiths effects in which rare fluctuations in the couplings of the Hamiltonian allow a long finite segment of the system to be "locally" in the "metal- lic" phase. A calculation of this contribution gives [9] a low-frequency conductivity $\sigma^{\prime}(\omega) \sim \omega^{\alpha} \ln ^{2}(\omega)$, where $\alpha>0$ is a continuously varying, nonuniversal exponent that vanishes at the transition.

The dynamical structure factor can be calculated in a similar way. Consider first $S^{z z}(k, \omega)$. One begins with the spectral sum (2). The leading order "operator renormalizations" needed in this case are particularly simple - each spin operator remains unchanged as long as it is not part of a singlet and renormalizes to zero upon being locked into a singlet state [11]. As before, we run the RG until the cutoff $\Omega=\omega$ and do the spectral sum with the renormalized operators in the new problem. This renormalized sum may be evaluated by again recognizing that it is dominated [12] by excitations to the triplet state $\left|t_{0}\right\rangle$ of pairs of spins connected by the renormalized bond $\tilde{J}^{\perp}=\omega$. The corresponding matrix element is simply $\left(1-e^{i k \tilde{l}}\right) / 2$, where $\tilde{l}$ is the length of the strong bond (note that $\tilde{l} / a$ is an odd integer). This allows us to write, for $\omega \ll \Omega_{c}$ and $k=\pi / a+q$,

$$
\begin{aligned}
S^{z z}(k, \omega) \sim & n_{\Omega_{\omega}} \int d l d J^{\perp}\left|1+e^{i q l}\right|^{2} \\
& \times \mathcal{P}\left(J^{\perp}, l \mid \omega\right) \delta\left(\omega-J^{\perp}\right)
\end{aligned}
$$

at all three RS fixed points. The calculation of $S^{ \pm}(k, \omega)$ is slightly more involved as the gap to the relevant triplet excited state (with $m^{z}=1$ ) of a pair of spins connected by a strong bond $\left(J^{\perp}, J^{z}\right)$ is now $\left(J^{\perp}+J^{z}\right) / 2$. However, a careful analysis [9] gives the same result as above for $\omega \ll \Omega_{c}$.

Let us focus here on the regime $|q| \equiv|k-\pi / a| \ll$ $a^{-1}$ (in addition to $\omega \ll \Omega_{c}$ ). In this regime, the integral can be evaluated using the results of Ref. [6] for $\mathcal{P}$. This gives the following rather unusual universal scaling form at the RS fixed points (i.e., as long as the ground state does not have IAF order):

$$
S^{\alpha \beta}(k, \omega)=\frac{\mathcal{A}}{\omega \ln ^{3}\left(\Omega_{c} / \omega\right)} \Phi\left(|q|^{1 / 2} \ln \left(\Omega_{c} / \omega\right) / v_{\zeta}^{1 / 2}\right),
$$

where $\alpha \beta \equiv+-$ or $z z, \mathcal{A}$, and $v_{\zeta}$ are nonuniversal scale factors, and the fully universal function $\Phi(x)$ can be written as

$$
\Phi(x)=\left(1+\frac{x[\cos (x) \sinh (x)+\sin (x) \cosh (x)]}{\cos ^{2}(x) \sinh ^{2}(x)+\sin ^{2}(x) \cosh ^{2}(x)}\right) .
$$

We now briefly summarize the effects of weak (compared to the disorder) enforced bond dimerization controlled by a dimensionless small parameter $|\delta|$ that sets the difference between the probability distributions of even and odd bonds [14]. The RG flows in the vicinity of the $X X$ and $X X X$-RS states are known $[6,8]$; the lowenergy properties are controlled by lines of strongdisorder random dimer [8] fixed points ending in the $X X$ and $X X X$ fixed points. These $\mathrm{RD}$ fixed points again 
describe ground states that consist of singlet pairs; however, now the singlet bonds preferentially start from an even (odd) site and end at an odd (even) site for positive (negative) $\delta$. Our approach readily allows us to follow the full crossover of the low-frequency dynamical conductivity and structure factor from the $X X$ and $X X X$-RS states to the corresponding RD phases (only the nonuniversal prefactors differ in the two cases). Here, we focus on results deep in the RD phases, i.e., for frequencies such that $\bar{\Gamma}_{\omega} \equiv|\delta| \ln \left(\Omega_{c} / \omega\right) \gg 1$. For the conductivity, we obtain $\sigma^{\prime}(\omega) \sim \ln \left(\Omega_{c} / \omega\right) \bar{\Gamma}_{\omega} e^{-c_{0} \bar{\Gamma}_{\omega}}$, which can be written more explicitly as $\sigma^{\prime}(\omega) \sim|\delta| \omega^{c_{0}|\delta|} \ln ^{2}\left(\Omega_{c} / \omega\right)$, where $c_{0}$ is a nonuniversal constant. The RD phases are thus seen to be gapless insulators. The dynamic structure factor in the vicinity of $k=\pi / a$ can be written as

$$
\begin{aligned}
S^{\alpha \beta}(k & \left.=\frac{\pi}{a}+q, \omega\right) \\
& =\frac{C|\delta|^{3}}{\omega^{1-\alpha}}\left[1+\cos \left(c_{1} \bar{\Gamma}_{\omega} \bar{q}\right) e^{-c_{2} \bar{\Gamma}_{\omega} \bar{q}^{2}}\right],
\end{aligned}
$$

where $\alpha=c_{0}|\delta|, c_{1}, c_{2}$, and $C$ are nonuniversal constants, and $\bar{q} \equiv q a / \delta^{2}$ is assumed $\ll 1$. This result has striking oscillatory structure which is best understood [9] as a novel signature of the sharply defined geometry of the rare Griffiths regions which contribute to the scattering at a given low frequency-more precisely, the average length of the relevant Griffiths regions is of order $\bar{\Gamma}_{\omega} a /|\delta|^{2}$ while the RMS fluctuations in the lengths are only of order $\sqrt{\bar{\Gamma}_{\omega}} a /|\delta|^{2}$.

In the spin-1 case, the specific Hamiltonian we consider is

$$
\mathcal{H}=\sum_{j} J_{j} \vec{S}_{j} \cdot \vec{S}_{j+1},
$$

where $\vec{S}_{j}$ are spin-1 operators and the $J_{j}$ are random, positive bond strengths; the corresponding distribution of $\ln \left(J_{j}\right)$ is characterized by a width $W$. A renormalization group analysis [7] reveals that the system flows to the ana$\log$ of the $X X X$-RS point described earlier only when $W$ exceeds a critical value $W_{c}$. In this case, our previous results for the dynamic structure factor and the dynamical conductivity continue to apply. As $W$ is decreased, the system undergoes a quantum phase transition to the so-called gapless Haldane (GH) phase [7]; both the quantum critical point and the GH phase in the vicinity of it are, however, still controlled by strong-disorder fixed points [7]. Our technique may also be used to calculate the dynamical conductivity at this critical point and in the GH phase, although the details differ considerably from the spin- $1 / 2$ case [9]. At the critical point, we find

$$
\sigma^{\prime}(\omega) \sim \ln ^{2}\left(\Omega_{c} / \omega\right),
$$

which is a stronger divergence than in the strongdisorder RS phase. In the GH phase, the conductivity goes as $\sigma^{\prime}(\omega) \sim \omega^{\alpha} \ln ^{2}\left(\Omega_{c} / \omega\right)$ for log-frequencies $\ln \left(\Omega_{c} / \omega\right) \gg\left(W_{c}-W\right)^{-\nu / 3}$ [ $\nu$ is the correlation length exponent known [7] to equal $6 /(\sqrt{13}-1)]$. This phase is thus a gapless insulator, not unlike the RD phase of spin- $1 / 2$ chains. The nonuniversal exponent $\alpha$ is given as $\alpha \sim\left(W_{c}-W\right)^{\nu / 3}$.

We close with some remarks regarding experiments. Our results for $S(k, \omega)$ near $k=\pi / a$ are clearly of direct relevance to low-temperature INS experiments on such spin-chain systems, especially in the regime where the energy transfer $\omega$ satisfies $T \lesssim \omega \ll \Omega_{c}$. Of particular interest would be an experimental confirmation of the coherent oscillatory structure predicted in the RD phase. Regarding transport, we hope that our results motivate experiments to probe the spin conductivity in these systems.

We thank P. W. Anderson, R. N. Bhatt, F. D. M Haldane, M. Hastings, A. Madhav, R. Moessner, S. Sachdev, T. Senthil, S.L. Sondhi, and A. Vishwanath for useful discussions. This work was supported by NSF Grants No. DMR-9809483 and No. DMR-9802468.

[1] C. Broholm et al., in Dynamical Properties of Unconventional Magnetic Systems, edited by A. Skjeltorp and D. Sherrington (Kluwer, Dordrecht, 1997).

[2] L. C. Tippie and W. G. Clark, Phys. Rev. B 23, 5846 (1981); Phys. Rev. B 23, 5854 (1981).

[3] C. P. Landee et al., Bull. Am. Phys. Soc. 44, 1942 (1999).

[4] C. Dasgupta and S. K. Ma, Phys. Rev. B 22, 1305 (1980).

[5] C. A. Doty and D. S. Fisher, Phys. Rev. B 45, 2167 (1992).

[6] D. S. Fisher, Phys. Rev. B 50, 3799 (1994); Phys. Rev. B 51, 6411 (1995).

[7] R. Hyman and K. Yang, Phys. Rev. Lett. 78, 1783 (1997); C. Monthus, O. Golinelli, and T. Jolicoeur, Phys. Rev. Lett. 79, 3254 (1997).

[8] R. Hyman et al., Phys. Rev. Lett. 76, 839 (1996).

[9] O. Motrunich, K. Damle, and D. A. Huse (to be published).

[10] R. N. Bhatt and P. A. Lee, Phys. Rev. Lett. 48, 344 (1982).

[11] Higher order corrections to the operator renormalization rules only affect various nonuniversal scale factors [9].

[12] This is a consequence of the extremely broad distributions of couplings in the low-energy effective theory.

[13] The free fermion Anderson localization problem that the $X X$ chain maps to is in a special universality class due to the presence of particle-hole symmetry, with a corresponding divergence of the localization length [L. Balents and M. P. A. Fisher, Phys. Rev. B 56, 12970 (1997), and references therein; H. Mathur, Phys. Rev. B 56, 15794 (1997)].

[14] We can also calculate [9] dynamical quantities for the random transverse field Ising chain [6] and compare with the past numerical work [H. Rieger and F. Igloi, Europhys. Lett. 39, 135 (1997); J. Kisker and A. P. Young, Phys. Rev. B 58, 14397 (1998)]. 